\title{
Hand preference for pointing gestures and bimanual manipulation around the vocabulary spurt period
}

\author{
Hélène Cochet, Marianne Jover, Jacques Vauclair* \\ Research Center in the Psychology of Cognition, Language, and Emotion, Department of Psychology, \\ Aix-Marseille University, 13621 Aix-en-Provence Cedex 1, France
}

\section{A R T I C L E I N F O}

\section{Article history:}

Received 12 January 2011

Revised 18 April 2011

Available online 24 May 2011

\section{Keywords:}

Language development

Lexical spurt

Handedness

Bimanual manipulation

Imperative pointing

Declarative pointing

\begin{abstract}
A B S T R A C T
This study investigated the development of hand preference for bimanual manipulative activities and pointing gestures in toddlers observed longitudinally over a 5-month period, in relation to language acquisition. The lexical spurt was found to be accompanied by an increase in the right-sided bias for pointing but not for manipulation. Moreover, results revealed a significant correlation between hand preference for imperative pointing gestures and manipulative activities in children who did not experience the lexical spurt during the observational period. By contrast, measures of handedness for declarative pointing were never correlated with those of handedness for manipulation. This study illustrates the complex relationship between handedness and language development and emphasizes the need to take the different functions of pointing gestures into account.
\end{abstract}

(c) 2011 Elsevier Inc. All rights reserved.

\section{Introduction}

A small body of research has described the development of hand preference in relation to language development, although this topic represents a significant source of information for assessing developmental changes of these two main functional asymmetries. Because the use of one hand for a specific activity mostly reflects the predominant involvement of the contralateral hemisphere, this measure provides an innovative means for investigating its relations with the cerebral control of speech during development. Moreover, different measurements of handedness may allow us to determine whether manipulative activities and communicative gestures are linked to speech at varying degrees. In the

\footnotetext{
* Corresponding author. Fax: +33442389170.

E-mail address: jacques.vauclair@univ-provence.fr (J. Vauclair).
} 
current study, language level and hand preference for both bimanual manipulation and pointing gestures were measured longitudinally in toddlers between 13 and 21 months of age in an attempt to unravel the complex relationships between language acquisition and the development of handedness. We focused on a key period of language development, namely the lexical spurt, whose onset was expected to be more closely linked to hand preference for pointing than to handedness for noncommunicative actions in the course of development. Moreover, speech-gesture relationships were expected to vary depending on the function served by pointing gestures.

\section{Development of handedness: manipulative activities}

Although the first signs of asymmetries emerge very early in the infant's development (see Provins, 1992, for a review) and are even already expressed in the fetus (e.g., Hepper, Shahidullah, \& White, 1990; Michel, 1981), the degree of hand preference is rather weak and fluctuating during the few months following the first intentional grasping movements, produced at around 5 months of age (e.g., Corbetta \& Thelen, 1999; Ramsay, 1985). Bimanual skills, emerging at around 1 year of age, are more likely to reveal stable indicators of handedness than unimanual activities such as reachto-grasp movements, and this is all the more true as the two hands play much differentiated roles (Fagard \& Lockman, 2005; Fagard \& Marks, 2000). For these bimanual activities, researchers deem the hand that plays an active role as the dominant hand and the one that is used as a passive support as the nondominant hand. This can be exemplified with the tube task, in which the nondominant hand grasps a tube while the dominant hand picks up the object or food inserted in it (e.g., Hopkins, 1995; Vauclair \& Imbault, 2009; Vauclair, Meguerditchian, \& Hopkins, 2005).

The most important cerebral processes related to the development of handedness are generally thought to take place before 3 years of age, resulting in an increase in the proportion of right-handed children for bimanual manipulative activities (Vauclair \& Imbault, 2009). However, another study with children observed at 13,20, and 28 months of age did not report any increase in the degree of manual asymmetry over this period (Bates, O'Connell, Vaid, Sledge, \& Oakes, 1986). Moreover, in assessing hand preference in a unimanual grasping task, Fagard and Marks (2000) observed an increase in the percentage of right-handers between 18 and 36 months of age. By contrast, the percentage of righthanders based on measures obtained from a bimanual coordination task in the same children was not found to vary, suggesting again that bimanual handedness is expressed earlier than unimanual handedness. However, the proportion of right-handed infants generally does not reach the $90 \%$ reported for adults (e.g., in infants: Esseily, Jacquet, \& Fagard, 2011; Michel, Sheu, Tyler, \& Ferre, 2006; in adults: Raymond \& Pontier, 2004). Therefore, although the direction of handedness appears to be stabilized at around 3 years of age, the strength of hand preference is likely to increase during later childhood until approximately 7 years of age (e.g., McManus et al., 1988).

In addition, methodological differences across studies complicate the issue of handedness development because there is a large variability in the criteria used to categorize individuals as right- or lefthanders and in the calculation of handedness indexes (see Hopkins, 1999). Thus, the understanding of the development of hand preference still needs to be improved, and longitudinal studies are particularly conducive to investigate this question.

\section{Gestural communication: pointing gestures}

Pointing is a referential and intentional communicative gesture that aims at indicating an object, an event, or a location to another person in a joint attentional frame (e.g., Camaioni, 1993). Pointing first emerges in human infants toward the end of their first year (e.g., Butterworth \& Morissette, 1996; Camaioni, Perucchini, Bellagamba, \& Colonnesi, 2004) and encompasses various functions and various forms (e.g., Cochet \& Vauclair, 2010a; Tomasello, Carpenter, \& Liszkowski, 2007). Researchers have mainly distinguished between imperative and declarative functions (Bates, Camaioni, \& Volterra, 1975), with the former being described as a request for a desired object or a specific action on that object and the latter being described as an attempt to direct the adult's attention to a referent in order 
to indicate its existence and share some interest in it (e.g., Camaioni, 1997). Thus, imperative pointing, at least in its early manifestations, may be regarded as an instrumental act that simply uses the adult as a means to a desired object (e.g., Bates et al., 1975). A few researchers have also argued that declarative pointing is used by infants as a means of gaining positive emotional reactions from the adult rather than to direct the attention of others to external entities (e.g., Moore \& Corkum, 1994). However, recent empirical findings, demonstrating that 12-month-old infants were able, first, to point cooperatively to provide information for other persons (e.g., Liszkowski, 2005) and, second, to request from adults absent but mutually known objects (e.g., Liszkowski, Schäfer, Carpenter, \& Tomasello, 2009) support the hypothesis that both imperative and declarative gestures reveal an early form of psychological understanding of others' mental states.

In addition to a lack of consensus concerning cognitive processes involved in the production of imperative and declarative gestures, it is also unclear whether these two kinds of pointing have distinct developmental trajectories and/or different relationships with speech development. Camaioni et al. (2004) observed that children were able to use imperative pointing earlier than declarative pointing; however, the opposite temporal shift has also been reported (Carpenter, Nagell, \& Tomasello, 1998). This discrepancy between studies might be explained by methodological differences because the production of pointing under experimental conditions (Camaioni et al., 2004) may differ from the production of pointing in joint attention episodes between mother and child observed in play situations (Carpenter et al., 1998). An observational study conducted in a day-care center revealed an age-related increase in the proportion of declarative pointing gestures produced by children between 1 and 3 years of age (Cochet \& Vauclair, 2010a), suggesting that children become more likely to declare about events and objects as they grow up. Moreover, declarative communicative gestures have been reported to be more tightly interconnected with the vocal system than are imperative gestures (e.g., Camaioni et al., 2004; Franco \& Butterworth, 1996). For example, declarative gestures are more frequently accompanied by vocalizations than are imperative gestures (Cochet \& Vauclair, 2010b). These different relations with speech between imperative and declarative gestures may be reflected in distinct hand preference patterns, as explained in the following section.

\section{Handedness for pointing and language development}

Speech-gesture links have been highlighted in many developmental studies, mainly pertaining to the predictive and facilitative effects of gestures on speech development (e.g., Butterworth, 2003; Iverson \& Goldin-Meadow, 2005; Pizzuto \& Capobianco, 2005; Rowe \& Goldin-Meadow, 2009; Tomasello, 2008). Moreover, from a neurobiological perspective, several researchers have postulated the existence of a relation between anatomical and functional hemispheric asymmetries associated with language and hand preference behavior (e.g., Hervé, Crivello, Perchey, Mazoyer, \& Tzourio-Mazoyer, 2006). Although left hemisphere specialization seems well established in righthanders (e.g., Knecht et al., 2000), relatively little is known concerning the exact nature of this relation and few data are available in human infants and children. Nonetheless, it has been argued that adult patterns of cerebral asymmetries are set early in infant development (e.g., Amunts, Schmidt-Passos, Schleicher, \& Zilles, 1997; Serrien, Ivry, \& Swinnen, 2006) and might even develop from processes controlling morphogenesis of the brain in the embryo (Trevarthen, 1996). Moreover, infants have been shown to exhibit left hemisphere lateralization in both speech perception (e.g., Dehaene-Lambertz, Dehaene, \& Hertz-Pannier, 2002; Mills, Coffey-Corina, \& Neville, 1993) and production. For example, a study using near-infrared spectroscopy in a 3-year-old child revealed clear left hemisphere activation in Broca's area during speech production (Gallagher et al., 2007).

Considering these early structural and functional hemispheric asymmetries in the speech-processing cerebral network (see also Dubois et al., 2009), it seems particularly relevant to investigate speech-gesture links through the development of hand preference for communicative gestures. A right-sided asymmetry especially for pointing gestures has been reported in several studies (Bates et al., 1986; Blake, O’Rourke, \& Borzellino, 1994; Vauclair \& Imbault, 2009; Young, Lock, \& Service, 1985). Moreover, Esseily et al. (2011) showed that right-handed infants for pointing understood and produced more words than non-right-handed infants, and Vauclair and Cochet (2010) observed 
a U-shaped relationship in toddlers between 12 and 30 months of age between the degree of hand preference for pointing and the developmental quotient for language. Results from the study by Bates et al. (1986) also support the existence of a dynamic nonlinear relationship between speech and righthand use: these authors failed to reveal significant correlations between language score and handedness in pointing and symbolic gestures, whereas a nonlinear relationship was observed at 20 months of age.

Thus, although the link between language and hand preference for pointing gesture seems quite obvious, little is currently known about the precise development of this relationship during ontogeny. Thus, a longitudinal study would appear to be appropriate to go some way toward answering this question, especially focusing on a key period of language development occurring during the second year of life, that is, the vocabulary spurt period. The lexical (or vocabulary) spurt is defined by an increase in lexical production occurring toward 18 months of age once children's vocabulary size reaches approximately 50 words (e.g., Goldfield \& Reznick, 1990; Nazzi \& Bertoncini, 2003). Such a strong increase in the rate of word learning has been suggested to trigger or reinforce the activation of analytical mechanisms (e.g., Locke, 1997) and/or to reflect a fundamental change in the word learning process (e.g., Behrend, 1990; Mervis \& Bertrand, 1994). These processes may be associated with a heavy demand placed on left hemisphere resources, which is more likely to highlight the tight interconnection in the brain between speech and communicative gestures. Besides, given the age ranges examined in the different studies mentioned earlier, it appears that a focus on the lexical spurt period may provide some explanations for the nonlinear relationships that were reported between hand preference for pointing and speech development.

In addition, because imperative and declarative pointing gestures seem to relate to speech to different degrees (see above), we may expect declarative pointing to be more right-handed than imperative pointing and/or to be more closely linked with the lexical spurt period. More precisely, hand preference for declarative gestures, but not imperative ones, may develop jointly with the increase in the rate of lexical growth characterizing the vocabulary spurt.

\section{Comparison between communicative gestures and non-communicative activities}

Even though studies of hand preference originally pertained to non-communicative object-directed actions, the distinction between communicative gestures and manipulative actions proves to be necessary because the comparison of these two types of activities may reveal different patterns of asymmetry, as well as different relationships with speech development. The right-sided bias has been shown to be stronger for infants' pointing gestures than for manipulative activities regardless of whether it concerns unimanual object grasping or bimanual manipulation (Bates et al., 1986; Cochet \& Vauclair, 2010b). Regarding correlational analyses, Esseily et al. (2011) did not observe any significant relationship between hand preference for pointing gesture and object grasping, although the majority of children were right-handed for both activities. Moreover, Vauclair and Imbault (2009), besides observing a higher number of right-handed participants in pointing than in object manipulation, reported a significant but moderate correlation between handedness scores for pointing gestures and object manipulation. More precisely, the correlation was significant between 18 and 20 months and between 29 and 32 months of age, and it became nonsignificant during the interim, which was interpreted by the authors as reflecting the influence of speech development on hand preference patterns. However, it is difficult to further explain these findings because language level was not directly measured in that study. Finally, few studies have compared hand preference patterns in communicative gestures and manipulative actions, and so far results tend to emphasize some independence between hand preferences for pointing gestures and manipulative activities.

Thus, using a longitudinal design over a 5-month period, this study aimed to explore the developmental patterns of handedness for both manipulative activities and pointing gestures during the second year of life in relation to the lexical spurt period. Another purpose of this study was to examine the difference between imperative and declarative pointing, notably with respect to their relationships with language development. 


\section{Method}

\section{Participants}

A total of 25 French children ( 13 girls and 12 boys), from Caucasian middle- to upper middle-class families, were studied once a month in day nurseries over a 5-month period. They were between 13 and 17 months of age at the first session. Among these participants, 11 ( 6 girls and 5 boys) took part in the study in 2008 and 14 ( 7 girls and 7 boys) took part in the study in 2009 (see Table 1). Children were considered to be 13 months old when their age ranged between 12.5 and 13.5 months and so forth for other ages in months. Parents provided informed consent for their infants' participation.

Because there were slight variations in the manipulation task and in the number of trials per task depending on the year of the experiment (2008 or 2009), we performed Mann-Whitney $U$ tests to compare the two groups of children for each variable of interest at each session. There was no significant difference in hand preference scores for either the manipulation task, the imperative pointing task, or the declarative pointing task. Moreover, no significant difference was observed in the language test score. Thus, data from the two groups of participants were combined for statistical analyses.

\section{Procedure}

Children had met the experimenters before the first day of the experiment to get familiarized with them. Each child was seated at a rectangular table, either in isolation in a separate room or in the main room but apart from the other children depending on the day-care center. One of the experimenters was sitting opposite the child, and the other experimenter stood back, noting the recorded behaviors. A bimanual manipulation task and two pointing tasks were administered, with the order of presentation alternated across participants. The two pointing tasks were designed to induce imperative and declarative pointing, respectively, based on earlier studies (Liszkowski et al., 2009; see also Blake et al., 1994, for a description of indicative and request situations). To avoid postural biases, data were recorded only when the child was in a symmetrical position with both hands initially free. Moreover, all of the objects and stimuli used were positioned centrally in front of the child. All sessions were videotaped.

\section{Bimanual manipulation}

Three variants of a bimanual task were administered, with the hand playing an active role being considered as the dominant hand and the hand having a role of support or orientation being considered as the nondominant hand. In the "bottle" variant, the child needed to hold a small transparent plastic bottle $(6 \mathrm{~cm}$ in diameter) with one hand and to take out the soft toy that was placed in it with the dominant hand. In the "sphere" variant, the participant needed to maintain a ball-shaped box (16 cm in diameter) with one hand and to put in a small ball $(3.5 \mathrm{~cm}$ in diameter) with the dominant hand or, on the contrary, to take that ball out. In the "column" variant, the child needed to remove a plastic ring from a Fisher Price column with the dominant hand by holding the base of the column with the other hand. According to the session (2008 or 2009), children performed either three trials of the sphere task and three trials of the column task or four trials of the sphere task and four trials of the bottle task. Thus, in total, children performed between six and eight trials.

Table 1

Distribution of participants depending on age at first session and year of experiment.

\begin{tabular}{lllllll}
\hline & 13 Months & 14 Months & 15 Months & 16 Months & 17 Months & Total \\
\hline Number of participants in 2008 & 0 & 3 & 2 & 5 & 1 & 11 \\
Number of participants in 2009 & 3 & 3 & 4 & 4 & 0 & 14 \\
Total & 3 & 6 & 6 & 9 & 1 & 25 \\
\hline
\end{tabular}


Imperative pointing gesture

The experimenter handled attractive toys (e.g., a wind-up ladybird) and showed interest about them, for example by saying, "Look at this! Isn't it funny?" She then put the object on the table, beyond the child's reach ( $\sim 50 \mathrm{~cm}$ away from the child), to induce a pointing gesture. When the child produced a pointing gesture, he or she was given the toy. If the child did not produce any gesture, the trial was considered as ended after approximately $10 \mathrm{~s}$. Children completed three or four trials according to the session (2008 or 2009). A new attractive toy was presented for each trial.

\section{Declarative pointing gesture}

For the declarative pointing task, we used piled up cubes, placed at a distance of $50 \mathrm{~cm}$ from the child, on which different drawings were stuck (e.g., a dog picture). The experimenter asked the child to show her the different pictures, for example by saying, "Have you seen the dog? Where is it?" When the child produced a pointing gesture, the experimenter commented about the picture. If the child did not produce any gesture, the trial was considered as ended after approximately $10 \mathrm{~s}$. This task aimed at leading children to direct the adult's attention to a picture in order to share some interest about it. Thus, children's gestures were taken into account even if the picture pointed at was different from the one first mentioned by the experimenter. Children performed three or four trials according to the session (2008 or 2009).

\section{Measures}

\section{Language}

To measure children's language level, parents were asked to fill out the French adaptation (Kern, 2003; Kern, 2007) of the MacArthur Communicative Development Inventories (MCDI) (Fenson et al., 1993) at every session. For the sake of comparison, we used a simplified version of the "Words and Sentences" questionnaire designed for children between 16 and 30 months of age. Only production was taken into account, with the score obtained corresponding to the total number of words children had in their vocabulary according to their parents. We removed one aberrant value for a 15-month-old participant because this outlier differed strikingly from the other data and likely resulted from a measurement error.

\section{Hand preference}

To assess hand use asymmetries, individual handedness index scores were calculated for each task with the formula $(R-L) /(R+L)$, where $R$ and $L$ stand for the total numbers of right- and left-hand responses, respectively. Handedness index values lay along a continuum from -1 to +1 , with the " \pm " sign indicating hand preference direction and the absolute value reflecting the strength of hand preference. Handedness indexes were calculated only when children had performed at least two trials.

Table 2

Number of observations depending on age and task.

\begin{tabular}{llllllllllr}
\hline & 13 & 14 & 15 & 16 & 17 & 18 & 19 & 20 & 21 & Total \\
& Months & Months & Months & Months & Months & Months & Months & Months & Months & \\
\hline $\begin{array}{c}\text { Language test } \\
\text { Bimanual }\end{array}$ & 2 & 6 & 12 & 18 & 18 & 17 & 13 & 10 & 1 & 97 \\
$\begin{array}{c}\text { manipulation } \\
\begin{array}{c}\text { Imperative } \\
\text { pointing }\end{array}\end{array}$ & 1 & 9 & 15 & 22 & 24 & 21 & 15 & 10 & 1 & 117 \\
$\begin{array}{c}\text { Declarative } \\
\text { pointing }\end{array}$ & 1 & 9 & 12 & 16 & 22 & 18 & 14 & 7 & 1 & 98 \\
\hline
\end{tabular}


Missing data

Due to children performing fewer than two trials in the pointing tasks or the manipulation task, or to some parents not always filling out the language questionnaire, some data were missing. The numbers of observations available depending on children's age and the task are presented in Table 2 .

\section{Results}

Preliminary Mann-Whitney $U$ tests did not reveal any gender effect on either handedness index scores or language test scores.

\section{Cross-sectional analysis}

Data were first examined cross-sectionally according to children's age. Because only 3 participants were 13 months old at the first session and only 1 participant was 21 months old at the last session, we removed these 4 observations and focused on the development between 14 and 20 months of age. The numbers of observation varied from 6 to 24 depending on the variable and the age considered. Wilcoxon signed ranks tests were used for age-related comparisons of language and handedness scores. Bonferroni corrections were applied to adjust the level of significance for these multiple comparisons $(p<.025)$.

\section{Language development}

Language scores ranged from 0 to 298 words and, not surprisingly, mean language scores increased between 14 and 20 months of age, as did interindividual variability (see Fig. 1). All of the two-by-two adjacent age differences were significant except the one between 14 and 15 months, $Z=1.6, p=.108$, $N=4$, which was performed on a limited number of children.

\section{Handedness development}

Mean handedness indexes (MHIs) associated with bimanual manipulation, imperative pointing gestures, and declarative pointing gestures are displayed in Table 3.

Two-by-two adjacent age comparisons did not reveal any significant difference in the mean handedness scores for either manipulation or pointing gestures in the course of development. Moreover, the comparison between bimanual manipulation and pointing gestures globally highlighted a stronger degree of hand preference for pointing gestures, although the difference was significant only at 18 months of age for declarative pointing, $Z=2.24, p<.025, N=21$. However, this age-related difference in MHIs remained minor and marginal. Thus, an analysis of handedness as a function of language development appears to be necessary for more significant patterns to emerge.

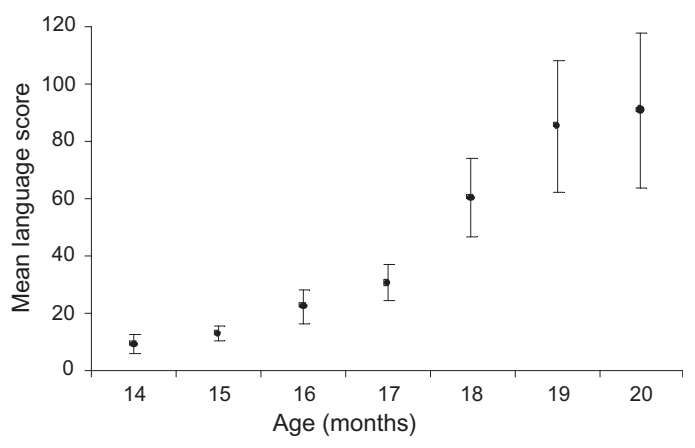

Fig. 1. Changes in mean language scores $( \pm \mathrm{SES})$ as a function of age. 
Table 3

Mean handedness indexes (and SEs) for the different manual activities at each age.

\begin{tabular}{llllllll}
\hline & 14 Months & 15 Months & 16 Months & 17 Months & 18 Months & 19 Months & 20 Months \\
\hline MHI bimanual & 0.21 & 0.42 & 0.52 & 0.49 & 0.54 & 0.39 & 0.67 \\
manipulation & $(0.23)$ & $(0.2)$ & $(0.12)$ & $(0.098)$ & $(0.14)$ & $(0.18)$ & $(0.14)$ \\
MHI imperative pointing & 0.43 & 0.75 & 0.81 & 0.39 & 0.55 & 0.76 & 0.71 \\
& $(0.37)$ & $(0.18)$ & $(0.11)$ & $(0.17)$ & $(0.15)$ & $(0.15)$ & $(0.29)$ \\
MHI declarative pointing & 0.37 & 0.41 & 0.77 & 0.64 & 0.9 & 0.73 & 0.56 \\
& $(0.16)$ & $(0.24)$ & $(0.14)$ & $(0.12)$ & $(0.048)$ & $(0.17)$ & $(0.29)$ \\
\hline
\end{tabular}

Note: Standard errors are in parentheses. MHIs usually vary from -1 to +1 ; a positive sign reflects right-hand preference, and the absolute value reflects the strength of hand preference.

\section{Handedness and speech development}

Spearman rank correlations did not reveal any significant relation between handedness indexes and language scores regardless of what activity and age were considered.

\section{Longitudinal analysis}

Subsequently, we investigated the relationship between laterality and language longitudinally, focusing on the lexical spurt period. The following analyses now include all of the observations from 13 to 21 months of age.

\section{Handedness and the lexical spurt}

To get a descriptive overview of the relationship between handedness and language development, all individual handedness indexes are depicted in scatter plots for the different manual activities. Figs. 2-4 suggest, first, the existence of different relationships between hand preference for manipulation, imperative pointing, and declarative pointing and the total number of words produced and, second, a qualitative change in these relations after the lexical spurt, that is, from the time children attained a 50-word productive vocabulary. Indeed, nearly all data points for which handedness indexes were negative were concentrated before the lexical spurt.

Among the 25 participants in the current study, 3 already produced more than 50 words at the time of the first session, 12 did not reach the 50 -word threshold associated with the onset of the lexical spurt, and 10 crossed this threshold during the observational period. The youngest children were

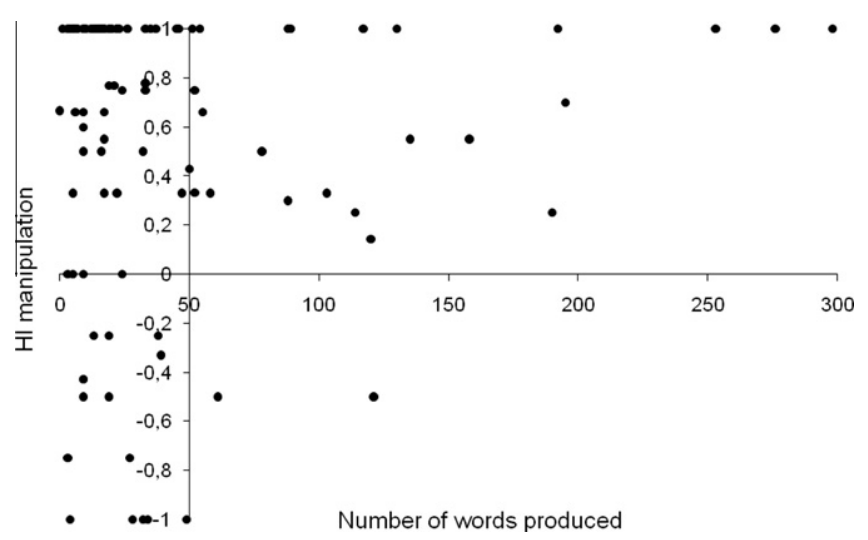

Fig. 2. Scatter plot displaying the relation between language score and handedness for manipulation. HI, handedness index. 


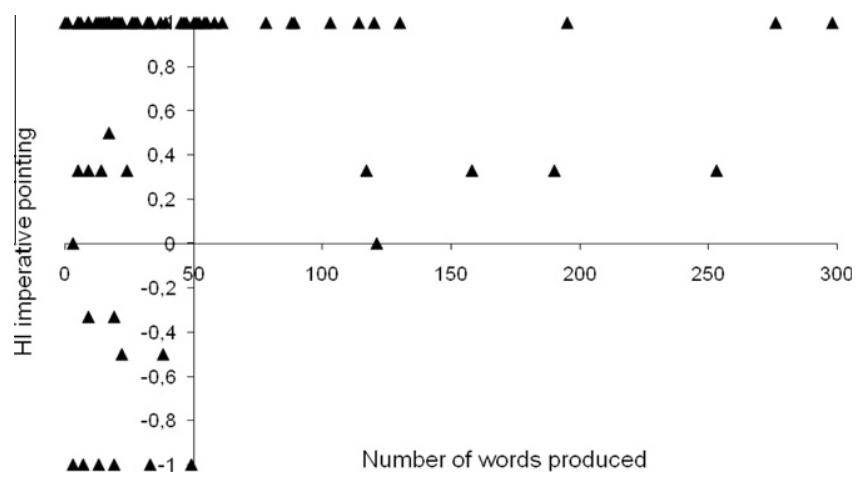

Fig. 3. Scatter plot displaying the relation between language score and hand preference for imperative pointing. HI, handedness index.

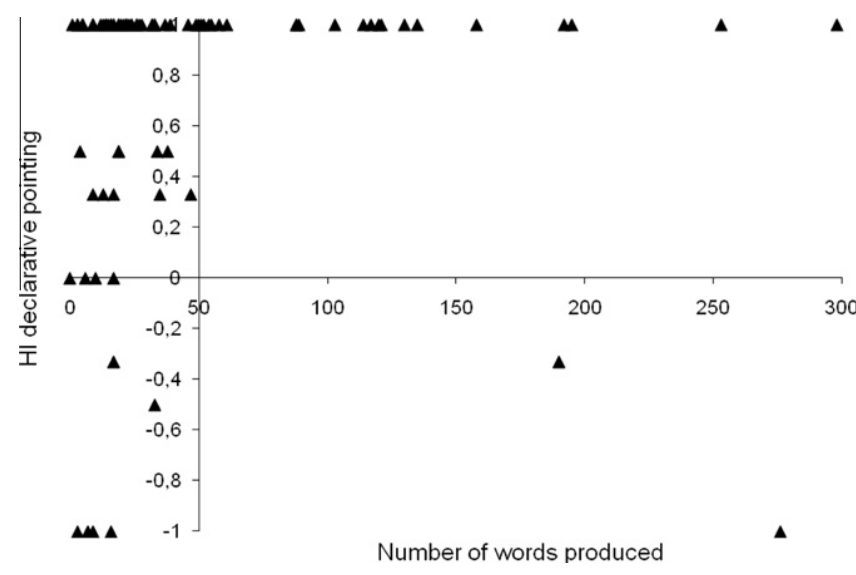

Fig. 4. Scatter plot displaying the relation between language score and hand preference for declarative pointing. HI, handedness index.

16 months old when their lexicon exceeded 50 words, and the oldest children were 20 months old $(M=18.1$ months, $S D=1.5)$.

First, Wilcoxon matched-pairs signed ranks tests and Spearman rank correlations were performed to examine the relation between handedness scores before and after the vocabulary spurt for each manual activity. Mean handedness scores, including only the children who reached the 50 -word threshold during the observational period, are displayed in Fig. 5. The MHI for imperative pointing gestures tended to be higher after the lexical spurt than before it, $Z=1.83, p=.068, N=9$, and the MHI for declarative pointing was higher after the lexical spurt, $Z=2.37, p<.05, N=9$. There was no difference in MHIs between these two periods for bimanual manipulation, $Z=0.085, n s, N=10$.

Second, we investigated the relationship between handedness scores recorded in the different tasks either before or after the lexical spurt. For the comparisons to be valid (i.e., performed on the same sample of children), analyses included only the participants who reached the 50 -word threshold during the observational period $(N=10)$.

\section{Pointing gestures}

There was no difference in MHIs between imperative and declarative pointing gestures either before the lexical spurt, $Z=0.17$, $n$, or after the lexical spurt, $Z=0.0, n s$. Moreover, handedness indexes for imperative and declarative gestures tended to be significantly correlated before the lexical spurt, 


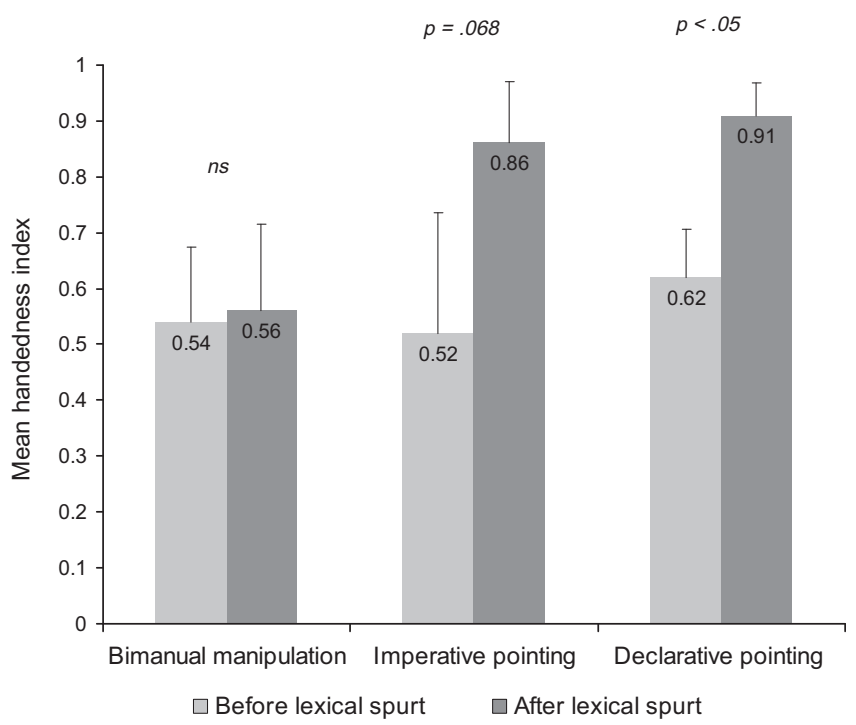

Fig. 5. Mean handedness indexes $( \pm S E s)$ for the different manual activities before and after the lexical spurt. Mean handedness indexes vary on a continuum from -1 to +1 ; a positive sign indicates right-hand preference, and the absolute value reflects the strength of hand preference. The level of significance associated with Wilcoxon matched-pairs signed ranks tests is indicated above the bars for each manual activity.

$R=.60, p=.067$, and were significantly correlated after the lexical spurt, $R=.96, p<.001$. These correlations were compared using Fisher's $Z$ transformation (see Raghunathan, Rosenthal, \& Rubin, 1996). The correlation between handedness indexes for imperative and declarative gestures proved to be stronger after the lexical spurt, $Z=2.05, p<.05$.

\section{Manipulation versus communication}

Before the lexical spurt, MHIs associated with imperative and declarative pointing gestures did not significantly differ from the MHI associated with bimanual manipulation, $Z=0.21, n s$, and $Z=0.53, n s$, respectively. By contrast, after the lexical spurt, the $\mathrm{MHI}$ for bimanual manipulation was found to be lower than MHIs associated with imperative and declarative pointing gestures, $Z=1.99, p<.05$. and $Z=2.02, p<.05$, respectively. Moreover, handedness indexes associated with imperative pointing gestures and bimanual manipulation were not significantly correlated either before the lexical spurt, $R=.46, n s$, or after the lexical spurt, $R=.44$, $n s$. Similarly, handedness indexes for declarative pointing were not correlated with handedness index for bimanual manipulation either before the lexical spurt, $R=.37, n s$, or after the lexical spurt, $R=.41, n s$.

Lastly, the following analyses focused on results obtained in children who did not reach the 50word threshold $(N=12)$.

\section{Pointing gestures}

There was no significant difference in MHIs between imperative and declarative pointing gestures, $Z=0.36$, ns. Moreover, handedness indexes for imperative and declarative gestures were not significantly correlated, $R=.46$, $n s$.

\section{Manipulation versus communication}

MHIs associated with imperative and declarative pointing gestures did not significantly differ from the MHI associated with bimanual manipulation, $Z=1.27, n s, N=22$, and $Z=0.36$, ns, respectively. 
Moreover, a significant correlation was found between hand preference for imperative gestures and manipulative activities, $R=.62, p<.05$. By contrast, handedness indexes for declarative pointing and bimanual manipulation were not significantly correlated, $R=.11, n s$.

To make sure that the previous results were not due to a general difference in the strength of hand preference between children who experienced the lexical spurt during the observational period and those who did not, we compared MHIs recorded before the lexical spurt between the two samples of children. Mann-Whitney $U$ tests did not reveal any difference between the two groups for either manipulative actions, $U=45.0, n s$, or pointing gestures, $U=52.5, n s$, for imperative pointing and $U=59.5$. $n s$, for declarative pointing.

To summarize, after the lexical spurt, hand preference associated with imperative and declarative pointing gestures was stronger than handedness for bimanual manipulation, whereas there was no difference between these measures before the lexical spurt. Moreover, in children who experienced the lexical spurt during the observational period of the current study, only the correlation between handedness indexes for imperative and declarative pointing was found to be significant. In children who did not experience the lexical spurt, handedness indexes for imperative and declarative gestures were not significantly correlated, whereas a significant correlation was observed between hand preference for imperative pointing gestures and manipulative activities.

\section{Discussion}

The main objective of the current study was to investigate the development of hand preference for communicative gestures and manipulative activities in relation to speech acquisition during the second year of life. First, a right-hand preference was observed for both bimanual manipulation and pointing gestures, confirming the results reported in several prior studies (e.g., Bates et al., 1986; Cochet \& Vauclair, 2010a, 2010b; Fagard \& Marks, 2000; Vauclair \& Imbault, 2009; Young et al., 1985).

Our results did not reveal any overall age-related increase in the right-sided bias for pointing gestures. Previous findings have not reported any strengthening of the right-sided asymmetry for pointing gestures between 13 and 28 months of age (Bates et al., 1986), between 10 and 40 months of age (Vauclair \& Imbault, 2009), and between 12 and 38 months of age (Cochet \& Vauclair, 2010a). By contrast, the degree of right-hand preference was found to augment between prepointing produced at 8 months of age and later pointing produced at 15 months (Young et al., 1985) and 12 months of age (Blake et al., 1994), likely reflecting an increasing involvement of the left cerebral hemisphere in the production of communicative gestures. Altogether, these results suggest that the right-sided asymmetry for communicative gestures is already strongly established by approximately 1 year of age.

Regarding bimanual manipulation, we also did not observe any age-related increase in the righthanded bias, in line with previous results reported in toddlers between 13 and 28 months of age (Bates et al., 1986) and between 18 and 36 months of age (Fagard \& Marks, 2000). Moreover, the overall increase in the proportion of right-handers for bimanual manipulative activities reported by Vauclair and Imbault (2009) was in fact due to a difference observed from 34 months of age onward. Thus, the strength of handedness may increase during early childhood (e.g., McManus et al., 1988) in a higher age range than the one selected for the current study.

Our results become more telling if we include language development in the picture. Indeed, toddlers' hand preference patterns were found to vary depending on whether or not the lexical spurt had taken place. This specific period in speech acquisition is characterized by a strong increase in the rate of word learning - children learn one or two new words a day - occurring at around 18 months of age. The age of onset of the vocabulary spurt varies sharply across children, but it has also been determined that lexical spurt occurs when children's productive vocabulary attains 50 words (Benedict, 1979; Goldfield \& Reznick, 1990; Nazzi \& Bertoncini, 2003; Nelson, 1973). We used this 50 -word milestone in the current study to contrast handedness scores as a function of the lexical spurt.

First, the comparison of hand preferences within each manual activity provided more information than the age-related analysis. There was no difference in handedness scores for bimanual 
manipulation between the two periods (before and after the lexical spurt). By contrast, the degree of hand preference for pointing gestures was found to be higher after the lexical spurt, although the difference was significant only for declarative pointing, whereas it tended to be significant for imperative pointing. Thus, even if a strong right-sided bias for pointing gestures is observed quite precociously, as stated above, this bias strengthens again as the lexical spurt takes place. This result suggests a tight interconnection in the left cerebral hemisphere between speech and communicative gestures, and this is especially true for declarative pointing, which is usually regarded as more closely related to speech development (e.g., Blake, Vitale, Osborne, \& Olshansky, 2005; Camaioni et al., 2004; Cochet \& Vauclair, 2010a). Moreover, the increase in the degree of hand preference for pointing reported toward the end of the first year (Blake et al., 1994; Young et al., 1985) might be associated with another important step in speech development that demands a high mobilization of left hemisphere resources, namely the production of the very first words.

The comparison between handedness scores for manipulation and communication also appears to be more meaningful taking the lexical spurt into account. Before that period, hand preference associated with imperative and declarative pointing gestures did not significantly differ from handedness associated with bimanual manipulation. By contrast, after the lexical spurt, hand preference was stronger for both imperative and declarative pointing than for bimanual manipulation. Moreover, handedness indexes for pointing gestures were not correlated with handedness indexes for bimanual manipulation. On the whole, these findings support the hypothesis of an independence between object manipulation and pointing gestures, associated with the idea that distinct neurobiological substrates in the left cerebral hemisphere control these behaviors (e.g., Vauclair \& Imbault, 2009). Considering the interaction between the production of pointing gesture and the lexical spurt, as well as the different functions of pointing, may allow us to clarify and develop this hypothesis.

Before the lexical spurt, the pace of word learning is quite slow and steady. The strong increase in the rate of word learning is likely to be coupled with an increasing involvement of the left cerebral hemisphere in linguistic processes, as suggested by an event-related potential study in 20-monthold infants (Mills et al., 1993). Once this cerebral network has reached a certain level of specialization - with the onset of the lexical spurt - the strength of hand preference for pointing gestures increases up to and exceeding the one for bimanual manipulation. Thus, we can raise the hypotheses that (a) the increasing cerebral specialization involves an integrated and bimodal communication system rather than just speech network and (b) the control of manipulative activities is independent from this communication system. These hypotheses are supported by recent neural evidence reporting simultaneous integration of information from speech and communicative gestures in the brain (Xu, Gannon, Emmorey, Smith, \& Braun, 2009; Özyürek, Willems, Kita, \& Hagoort, 2007). This left-lateralized and modality-independent system is likely to be located in Broca's area (Gentilucci \& Dalla Volta, 2007).

Interestingly, hand preference scores for imperative pointing were significantly correlated with measures of handedness for object manipulation in children who did not experience the vocabulary spurt, that is, in children who did not reach the 50-word threshold during the observational period. Moreover, among the same children, handedness indexes for imperative and declarative gestures were not significantly correlated. These results, which support the distinction between imperative and declarative gestures, may reflect the more instrumental and object-related nature of imperative pointing (Camaioni, 1997). Children produce imperative pointing to obtain something for themselves, and in their early manifestations, imperative pointing gestures may rely on a child's understanding of the other person as a causal agent. A shift in the cognitive abilities associated with imperative pointing has previously been suggested as children grow older (Tomasello et al., 2007) together with a change in the children's real intention. It was hypothesized that at an early stage, infants aim to influence the adult's behavior, whereas later on, as the adult comes to be regarded as an intentional agent who can decide to help the children, the latter may seek to influence the adult's goals and attention (Cochet \& Vauclair, 2010a). The current study enables us to highlight the period in speech development that is associated with this gradual shift, namely the lexical spurt. In agreement with our interpretation, Nazzi and Bertoncini (2003) proposed that this period corresponds to the onset of the referential use of language resulting from a developmental coupling of linguistic and cognitive abilities. The lexical spurt has also been suggested to 
be associated with a shift toward more analytical processes (e.g., Behrend, 1990; Locke, 1997; Mervis \& Bertrand, 1994). In contrast, McMurray (2007) argued that the lexical spurt is not related to any specialized learning processes but rather is a by-product of variation in difficulty; that is, the number of words likely to be to learned by children increases with the level of difficulty. The existence of the lexical spurt has also been questioned by some researchers for whom the increase in the rate of word learning is usually more gradual than has been assumed (e.g., Ganger \& Brent, 2004). Nonetheless, although the lexical spurt might not occur in all children or may happen later in some children than in others, a recent study showed that the growth rate of lexical production increased during the second year in most children (e.g., Stolt, Haataja, Lapinleimu, \& Lehtonen, 2008). Beyond the debate on the lexical spurt, the fact remains that in the current study handedness patterns changed when children reached a certain level of language development $( \pm 50$ words).

Contrary to the results concerning imperative gestures, measures of hand preference for declarative gestures were not correlated with those of handedness for manipulation in any of the children's groups. It has previously been argued that declarative pointing, produced with the aim of engaging with the adult and sharing interest in a specific object or event, reflects some infants' understanding of others' psychological states (e.g., Camaioni et al., 2004; Liszkowski, Carpenter, Henning, Striano, \& Tomasello, 2004). This early form of social understanding has been demonstrated in 12-month-old infants, when declarative pointing has just emerged (Liszkowski, Carpenter, \& Tomasello, 2007), consistent with our results showing that hand preference for declarative pointing is associated from the beginning with hemispheric asymmetries in communicative functions and not with asymmetries in purely motor functions of manipulation.

Thus, our findings suggest that imperative pointing and declarative pointing involve different levels of social understanding during the first few months of the second year of life. However, these gestures, as intentional and communicative signals, remain closely related. There was indeed no difference in the degree of hand preference between imperative and declarative pointing gestures, and these measures were significantly correlated, in line with previously reported results (Cochet \& Vauclair, 2010b). However, this correlation was stronger after the lexical spurt (Spearman correlation coefficient increased from .60 to .96) and was not significant in children who did not cross the 50-word threshold during the observational period, supporting the hypothesis of a qualitative change of imperative pointing once the lexical spurt has occurred.

Finally, the results of the current study may explain some discrepancies between studies that have shown, on the one hand, significant but moderate correlations between handedness indexes for manipulative actions and pointing gestures (Vauclair \& Imbault, 2009) and, on the other, the absence of any significant correlation between these two measures (Cochet \& Vauclair, 2010b). The investigation of hand preference in relation to the occurrence of lexical spurt may have revealed different and finer patterns.

It is also important to mention certain limitations of the current study, most of which are inherent in longitudinal designs. In addition to a relatively small sample size, the numerous missing data did not allow us to use a more analytical approach when analyzing the results (e.g., growth modeling, generalized estimating equation [GEE] analysis). Thus, larger samples should be examined in further research studies, possibly expanding the age range of the children beyond 21 months given that some participants of the current study were probably still too young to experience the lexical spurt.

In conclusion, the investigation of hand preference patterns, even as indirect indexes of hemispheric activity, has highlighted the relation between speech acquisition and declarative pointing gestures. Our results support the existence of a bimodal communication system in the left cerebral hemisphere that is different from the one involved in object manipulation. The current study also emphasized that the production of imperative pointing was associated with this manipulation system during the first few months of the second year of life before being more closely related to the communication system with the onset of the lexical spurt. Thus, in future research investigating the relationship between language development and handedness, it appears to be essential to consider the period of the vocabulary spurt and to distinguish between the imperative and declarative functions of pointing. 


\section{Acknowledgments}

The authors thank Floriane Cheneval, Radia Dhafeur, Pauline Léturgie, and Marion Pams for their help in collecting the data. This research was supported by a French National Research Agency (ANR) grant (ANR-08-BLAN-0011_01).

\section{References}

Amunts, K., Schmidt-Passos, F., Schleicher, A., \& Zilles, K. (1997). Postnatal development of interhemispheric asymmetry in the cytoarchitecture of human area 4. Anatomy and Embryology, 196, 393-402.

Bates, E., Camaioni, L., \& Volterra, V. (1975). The acquisition of performatives prior to speech. Merrill-Palmer Quarterly, 21, 205-226.

Bates, E., O'Connell, B., Vaid, J., Sledge, P., \& Oakes, L. (1986). Language and hand preference in early development. Developmental Neuropsychology, 2, 1-15.

Behrend, D. A. (1990). The development of verb concepts: Children's use of verbs to label familiar and novel events. Child Development, 61, 681-696.

Benedict, H. (1979). Early lexical development: Comprehension and production. Journal of Child Language, 6, 183-200.

Blake, J., O'Rourke, P., \& Borzellino, G. (1994). Form and function in the development of pointing and reaching gestures. Infant Behavior and Development, 17, 195-203.

Blake, J., Vitale, G., Osborne, P., \& Olshansky, E. (2005). A cross-cultural comparison of communicative gestures in human infants during the transition to language. Gesture, 5, 201-217.

Butterworth, G., \& Morissette, P. (1996). Onset of pointing and the acquisition of language in infancy. Journal of Reproductive and Infant Psychology, 14, 219-231.

Camaioni, L. (1993). The development of intentional communication: A reanalysis. In J. Nadel \& L. Camaioni (Eds.), New perspectives in early communicative development (pp. 82-96). London: Routledge.

Camaioni, L. (1997). The emergence of intentional communication in ontogeny, phylogeny, and pathology. European Psychologist, 2, 216-225.

Camaioni, L., Perucchini, P., Bellagamba, F., \& Colonnesi, C. (2004). The role of declarative pointing in developing a theory of mind. Infancy, 5, 291-308.

Carpenter, M., Nagell, K., \& Tomasello, M. (1998). Social cognition, joint attention, and communicative competence from 9 to 15 months of age. Monographs of the Society for Research in Child Development, 63(4), 1-143.

Cochet, H., \& Vauclair, J. (2010a). Features of spontaneous pointing gestures in toddlers. Gesture, 10(1), 86-107.

Cochet, H., \& Vauclair, J. (2010b). Pointing gestures produced by toddlers from 15 to 30 months: Different functions, hand shapes, and laterality patterns. Infant Behavior and Development, 33, 432-442.

Corbetta, D., \& Thelen, E. (1999). Lateral biases and fluctuations in infants' spontaneous arm movements and reaching. Developmental Psychobiology, 34, 237-255.

Dehaene-Lambertz, G., Dehaene, S., \& Hertz-Pannier, L. (2002). Functional neuroimaging of speech perception in infants. Science, 298, 2013-2015.

Dubois, J., Hertz-Pannier, L., Cachia, A., Mangin, J. F., Le Bihan, D., \& Dehaene-Lambertz, G. (2009). Structural asymmetries in the infant language and sensori-motor networks. Cerebral Cortex, 19, 414-423.

Esseily, R., Jacquet, A. Y., \& Fagard, J. (2011). Handedness for grasping objects and pointing and the development of language in 14-month-old infants. Laterality. doi:10.1080/1357650X.2010.499911.

Fagard, J., \& Lockman, J. (2005). The effect of task constraints on infants' (bi)manual strategy for grasping and exploring objects. Infant Behavior and Development, 28, 305-315.

Fagard, J., \& Marks, A. (2000). Unimanual and bimanual tasks and the assessment of handedness in toddlers. Developmental Science, 3, 137-147.

Fenson, L., Dale, P., Reznick, S., Thal, D., Bates, E., Hartung, J., et al (1993). MacArthur Communicative Development Inventories: User's guide and technical manual. San Diego: Singular Publishing Group.

Franco, F., \& Butterworth, G. (1996). Pointing and social awareness: Declaring and requesting in the second year. Journal of Child Language, 23, 307-336.

Gallagher, A., Theriault, M., Maclin, E., Low, K., Gratton, G., Fabiani, M., et al (2007). Near-infrared spectroscopy as an alternative to the Wada test for language mapping in children, adults, and special populations. Epileptic Disorder, 9, 241-255.

Ganger, J., \& Brent, M. R. (2004). Reexamining the vocabulary spurt. Developmental Psychology, 40, 621-632.

Gentilucci, M., \& Dalla Volta, R. (2007). The motor system and the relationships between speech and gesture. Gesture, 7 , $159-177$.

Goldfield, B. A., \& Reznick, S. J. (1990). Early lexical acquisition: Rate, content, and the vocabulary spurt. Journal of Child Language, 17, 171-183.

Hepper, P. G., Shahidullah, S., \& White, R. (1990). Origins of fetal handedness. Nature, 347, 431.

Hervé, P. Y., Crivello, F., Perchey, G., Mazoyer, B., \& Tzourio-Mazoyer, N. (2006). Handedness and cerebral anatomical asymmetries in young adult males. NeuroImage, 29, 1066-1079.

Hopkins, W. D. (1995). Hand preferences for a coordinated bimanual task in 110 chimpanzees: Cross-sectional analysis. Journal of Comparative Psychology, 109, 291-297.

Hopkins, W. D. (1999). On the other hand: Statistical issues in the assessment and interpretation of hand preference data in nonhuman primates. International Journal of Primatology, 20, 851-866.

Iverson, J. M., \& Goldin-Meadow, S. (2005). Gesture paves the way for language development. Psychological Science, 16, $367-371$.

Kern, S. (2003). Le compte rendu parental au service de l'évaluation de la production lexicale des enfants français entre 16 et 30 mois [Evaluation through parental report of lexical development in French children from 16 to 30 months]. Glossa, 85, 48-61. 
Kern, S. (2007). Lexicon development in French-speaking infants. First Language, 27, 227-250.

Knecht, S., Deppe, M., Dräeger, B., Bobe, L., Lohmann, H., Ringelstein, E. B., et al (2000). Language lateralization in healthy righthanders. Brain, 123, 74-81.

Liszkowski, U. (2005). Human twelve-month-olds point cooperatively to share interest with and helpfully provide information for a communicative partner. Gesture, 5(1-2), 135-154.

Liszkowski, U., Carpenter, M., Henning, A., Striano, T., \& Tomasello, M. (2004). Twelve-month-olds point to share attention and interest. Developmental Science, 7, 297-307.

Liszkowski, U., Carpenter, M., \& Tomasello, M. (2007). Pointing out new news, old news, and absent referents at 12 months of age. Developmental Science, 10, 1-7.

Liszkowski, U., Schäfer, M., Carpenter, M., \& Tomasello, M. (2009). Prelinguistic infants, but not chimpanzees, communicate about absent entities. Psychological Science, 20, 654-660.

Locke, J. L. (1997). A theory of neurolinguistic development. Brain and Language, 58, 265-326.

McManus, I. C., Sik, G., Cole, D. R., Mellon, A. F., Wong, J., \& Kloss, J. (1988). The development of handedness in children. British Journal of Developmental Psychology, 6, 257-273.

McMurray, B. (2007). Defusing the childhood vocabulary explosion. Science, 317, 631

Mervis, C. B., \& Bertrand, J. (1994). Acquisition of the novel name nameless category (N3C) principle. Child Development, 65 , 1646-1663.

Michel, G. F. (1981). Right-handedness: A consequence of infant supine head-orientation? Science, 212, 685-687.

Michel, G. F., Sheu, C. F., Tyler, A. N., \& Ferre, C. L. (2006). The manifestation of infant hand-use preferences when reaching for objects during the seven- to thirteen-month age period. Developmental Psychobiology, 48, 436-443.

Mills, D. L., Coffey-Corina, S. A., \& Neville, H. J. (1993). Language acquisition and cerebral specialization in 20-month-old infants. Journal of Cognitive Neuroscience, 5, 317-334.

Moore, C., \& Corkum, V. (1994). Social understanding at the end of the first year of life. Developmental Review, 14, 349-372.

Nazzi, T., \& Bertoncini, J. (2003). Before and after the vocabulary spurt: Two modes of word acquisition? Developmental Science, $6,136-142$.

Nelson, K. (1973). Structure and strategy in learning to talk. Monographs of the Society for Research in Child Development, 38(1-2, Serial No. 149).

Özyürek, A., Willems, R. M., Kita, S., \& Hagoort, P. (2007). On-line integration of semantic information from speech and gesture: Insights from event-related brain potentials. Journal of Cognitive Neuroscience, 19, 605-616.

Pizzuto, E., \& Capobianco, M. (2005). The link and differences between deixis and symbols in children's early gestural-vocal system. Gesture, 5(1), 179-199.

Provins, K. A. (1992). Early infant asymmetries and handedness: A critical evaluation of the evidence. Developmental Neuropsychology, 8, 325-365.

Raghunathan, T. E., Rosenthal, R., \& Rubin, D. B. (1996). Comparing correlated but nonoverlapping correlations. Psychological Methods, 1, 178-183.

Ramsay, D. S. (1985). Fluctuations of unimanual hand preference in infants following the onset of duplicated syllable babbling. Developmental Psychology, 21, 318-324.

Raymond, M., \& Pontier, D. (2004). Is there geographical variation in human handedness? Laterality, 9, 35-52.

Rowe, M. L., \& Goldin-Meadow, S. (2009). Early gesture selectively predicts later language learning. Developmental Science, 12, 182-187.

Serrien, D. J., Ivry, R. B., \& Swinnen, S. P. (2006). Dynamics of hemispheric specialization and integration in the context of motor control. Nature Reviews Neuroscience, 7, 160-166.

Stolt, S., Haataja, L., Lapinleimu, H., \& Lehtonen, L. (2008). Early lexical development of Finnish children: A longitudinal study. First Language, 28, 259-279.

Tomasello, M. (2008). Origins of human communication. Cambridge, MA: MIT Press.

Tomasello, M., Carpenter, M., \& Liszkowski, U. (2007). A new look at infant pointing. Child Development, 78, 705-722.

Trevarthen, C. (1996). Lateral asymmetries in infancy: Implications for the development of the hemispheres. Neuroscience and Biobehavioral Reviews, 20, 571-586.

Vauclair, J., \& Cochet, H. (2010). Laterality for pointing and language development in toddlers. Aix-Marseille University: Unpublished manuscript.

Vauclair, J., \& Imbault, J. (2009). Relationships between manual preferences for manipulative actions and for pointing gestures in infants and toddlers. Developmental Science, 12, 1060-1069.

Vauclair, J., Meguerditchian, A., \& Hopkins, W. D. (2005). Hand preferences for unimanual and coordinated bimanual tasks in baboons (Papio anubis). Cognitive Brain Research, 25, 210-216.

Xu, J., Gannon, P. J., Emmorey, K., Smith, J. F., \& Braun, A. R. (2009). Symbolic gestures and spoken language are processed by a common neural system. Proceedings of the National Academy of Sciences of the United States of America, 106, $20664-20669$.

Young, A. M., Lock, A. J., \& Service, V. (1985). Infants' hand preferences for actions and gestures. Developmental Neuropsychology, $1,17-27$. 